\title{
Short Communication: A 24 Hour Ecotoxicity Test for Acid Mine Drainage Using Hatching Success in Daphnia magna
}

\section{EBENEZER ALUMA*, R. PATRICK HASSETT AND KELLY JOHNSON}

\author{
Department of Biological Sciences, Ohio University, Athens, OH, USA 45701
}

\begin{abstract}
A bioassay utilizing the cladoceran Daphnia magna hatching success is presented for testing the toxicity of acid mine drainage (AMD) sediments. AMD sediments contain complex mixtures of potential toxins and a rapid, sensitive bioassay is valuable for assessing toxicity in the field, particularly after transient environmental events. Daphnia magna was incubated in water exposed to 1. clean sand spiked with different proportions of a mixture of metal salts and 2. sand mixed with different proportions of AMD sediments. Hatching success was linearly related to both the proportion of artificially spiked sediments $(r=-0.83 ; p=0.0002)$ and the proportion of AMD sediments $(\mathrm{r}=-0.74 ; \mathrm{p}=0.02)$ that Daphnia magna was exposed to during incubations. No animals hatched during the first $18 \mathrm{hrs}$ of observation under any treatments, so a single observation at $24 \mathrm{~h}$ is practical for this assay. The assay should be generally applicable to toxicity testing, particularly when assessing complex mixtures of pollutants in field settings. The novelty of the proposed study lies in the fact that it proposes the fastest ecotoxicity assay that we know of using Dahpnia magna. Potentially, after appropriate calibration for the particular pollutant of interest, it could be used to test for a wide array of aquatic pollutants. @ JASEM
\end{abstract}

Acid mine drainage (AMD) is a common environmental problem in areas with a history of coal mining. AMD develops when pyrite stores from disused coal mines come into contact with water (from rain events or nearby streams), leading to oxidation reactions that yield ferrous iron $\left(\mathrm{Fe}^{2+}\right)$, Sulfate $\left(\mathrm{SO}_{4}{ }^{2-}\right)$ and acidity as hydrogen ions $\left(\mathrm{H}^{+}\right)$. The sulfuric acid and hydrogen ions lower the $\mathrm{pH}$ and cause the mobilization of other heavy metals such as (Al, $\mathrm{Mn}, \mathrm{Zn}, \mathrm{Pb}, \mathrm{Cu}, \mathrm{As}, \mathrm{Ni}, \mathrm{Cd}, \mathrm{Co}$ ) from exposed coal or bedrock (Smith and Shumate, 1971; Johnson and Thornton, 1987). Because AMD is a complex assortment of pollutants that varies spatially and temporally, characterizing the severity of AMD impact is challenging. The $24 \mathrm{~h}$ ecotoxicity test proposed here uses hatching success in Daphnia magna to provide a rapid and sensitive test of the toxicity of acid-mine drainage sediments.

Daphnia magna, a pelagic cladoceran found in ephemereal pools and small water bodies, is widely used in aquatic ecotoxicology testing (Sobral et al., 2001; Lopes and Ribeiro 1999; Pereira et al., 2000). Reasons for the popularity of Daphnia magna in ecotoxicity testing include its relatively short life cycle, high fecundity and ease of culture and handling in the laboratory (Baudo, 1987). The sensitivity of this organism to pollution makes it valuable as an indicator of pollution. Various organizations, including the USEPA, OECD and ASTM, have developed standardized protocols for toxicity tests using
Daphnia magna (EPA, 2000; ASTM, 2006). Tests that utilize larval/embryonic stages are typically more sensitive than those that utilize adult organisms (Dave 1993). Protocols utilizing Daphnia magna developmental responses range in duration from the classic 21 day test to the 68 $\mathrm{h}$ test proposed by Sobral et al., (2001). Swimming-inhibition tests (reviewed by Martins et al. 2007) are relatively rapid (24h or $48 \mathrm{~h}$ ) but are performed on adults. However, short-term tests such as the swimming inhibition test are particularly valuable in the detection and management of transient pollution events, Therefore we have developed a $24 \mathrm{~h}$ test that combines the advantages of brief duration with the sensitivity of using developmental stages of Daphnia. Sobral et al. (2001) used three developmental stages of Daphnia magna as their endpoints; Daphnia produces homogenous brown eggs that are passed from its ovary to its brood chamber. Subsequently, (about 30h after the eggs arrive in the brood chamber) there is a visible separation of the external and internal egg membranes (stage one). The second stage is the differentiation of the head and body regions, the formation of rudimentary antennae and the appearance of two pink eyes in the head region (about 48 hours after arrival in the brood chamber). Further development results in the fusion of the two eyes to form a single median black eye, the release of a cuticle over the feeding apparatus and increased mobility of the antennae (about 68 hours after arrival in the brood chamber). This is the third stage which 
marks the end of egg development and the neonate is able to exist independently at this point (Sobral et al., 2001). The test proposed here determines hatching success of neonates identified at the single median eye stage that have been exposed to contaminated sediments during $24 \mathrm{~h}$ incubation.

\section{MATERIAL AND METHODS}

A stock culture of Daphnia magna was obtained from Ward Scientific Inc. On arrival in the laboratory, the organisms were placed in twenty gallons of culture medium at temperatures between $20^{\circ} \mathrm{C}$ and $23.5^{\circ} \mathrm{C}$ and left to acclimate for 24 hours. Culture medium was made using de-ionized water through which air had been bubbled for 24 hours. At the end of the 24 hour period, 1 teaspoonful of R/O Right ${ }^{\circledR}$ (Kent Marine Inc, Acworth, GA) was added to every 10 gallons of the aerated de-ionized water, to ensure $>25 \mathrm{ppm} \mathrm{CaCO}_{3}$ hardness. After acclimatization, the animals were placed in three tanks, each containing 10 gallons of medium, with gentle aeration and fed six drops of Rotirich $^{\circledR}$ (Florida Aqua Farms Inc., Dade City, FL ) per tank, every other day. The tanks were maintained at a temperature between $20^{\circ} \mathrm{C}$ and $23.5^{\circ} \mathrm{C}$, away from direct sunlight. The light regime was approximately 16 daylight hours: 8 dark hours as in Sobral et al. (2001). At weekly intervals, debris was siphoned from the bottom of the tanks using a turkey baster. At bi-weekly intervals, the medium was siphoned off and replaced to prevent fouling. The tanks were also sub-cultured at bi-weekly intervals to ensure continued production of parthenogenic eggs and to prevent overcrowding or the production of males and ephippia. After every brood hatched, we moved parent organisms to other tanks so that each tank contained individuals of the same age (and size class). In this way, we minimized hatching success variability due to age differences.

Four $300 \mathrm{ml}$ Petri dishes were prepared, each containing $2 \mathrm{~g}$ of uncontaminated Kolorscape ${ }^{\circledR}$ white play sand and $300 \mathrm{ml}$ of culture medium. Ten gravid Daphnia females with single "median eye" eggs that had been previously counted were placed in each Petri dish. Egg counting was done using a Nikon SMZ-2T microscope. The Petri dishes were monitored every six hours for neonates.

Spiked sediment was prepared by mixing $200 \mathrm{~g}$ of Kolorscape ${ }^{\circledR}$ white play sand with a mixture of metallic salts comprising $0.076 \mathrm{~g}$ each of zinc chloride, ferric chloride hexahydrate, manganese sulfate monohydrate and cupric chloride dehydrate (Sigma Chemical Co., St. Louis Missouri). The metallic salt mixture was thoroughly mixed with the Kolorscape ${ }^{\circledR}$ sand and placed in a 1 liter 1 beaker. $800 \mathrm{ml}$ of deionized water was added to spiked sand and stirred. The choice of water to sand ratio was based on D'sa (2004). The beaker containing the sand and water mixture was then oven-dried at $60^{\circ} \mathrm{C}$ for 48 hours, by which all the liquid had evaporated from the beaker. The residual sand was then thoroughly mixed. This spiked sand was then stored in a polythene bag until its use (Martinez-Tabche et al., 2000). Eleven concentrations of spiked sediment were prepared by mixing varying proportions of spiked sand in the preceding section, with clean Kolorscape ${ }^{\circledR}$ white play sand (Table 1). After thorough mixing of the spiked and clean sand, two grams of each concentration of contaminated sand was placed in $300 \mathrm{ml}$ Petri dishes. Each concentration was set up in triplicate so that there were 33 Petri dishes containing contaminated sand. A control experiment was also set up in three replicates using $2 \mathrm{~g}$ of uncontaminated sand in placed in $300 \mathrm{ml}$ Petri dishes. $300 \mathrm{ml}$ of culture medium was added to each Petri dish and the Petri dishes were left to equilibrate for $48 \mathrm{~h}$. After equilibration, 10 gravid female individuals were placed in each Petri dish and the dishes were checked at $6 \mathrm{~h}$ intervals.

\begin{tabular}{cll} 
Table 1. Proportion of spiked and clean sand used in bioassay. \\
\hline Concentration & Spiked sand $(\mathbf{g})$ & Clean sand $(\mathbf{g})$ \\
$\mathbf{1}$ & 1.00 & 1.00 \\
$\mathbf{2}$ & 0.50 & 1.50 \\
$\mathbf{3}$ & 0.25 & 1.75 \\
$\mathbf{4}$ & 0.12 & 1.88 \\
$\mathbf{5}$ & 0.10 & 1.90 \\
$\mathbf{6}$ & 0.09 & 1.91 \\
$\mathbf{7}$ & 0.08 & 1.92 \\
$\mathbf{8}$ & 0.07 & 1.93 \\
$\mathbf{9}$ & 0.06 & 1.94 \\
$\mathbf{1 0}$ & 0.05 & 1.95 \\
$\mathbf{1 1}$ & 0.02 & 1.98 \\
control & 0 & 2.00 \\
\hline
\end{tabular}


Sediment was collected from an acid mine drainage (AMD) seepage in Murray City, Ohio. This sediment was dried as described above. 10 concentrations of AMD sediments were prepared by mixing known quantities of dried AMD sediment with clean Kolorscape ${ }^{\circledR}$ play sand. Each concentration was done in three replicates. A control experiment was similarly set up in three replicates. Ten gravid Daphnia magna females, whose embryos were at the single median eye stage and whose eggs had been previously counted were placed in each Petri dish. Three Petri dishes containing $2 \mathrm{~g}$ of clean sand and $300 \mathrm{ml}$ of culture medium were designated as control experiments. The Petri dishes were checked at 12 hour intervals for successfully hatched embryos.

\section{RESULTS AND DISCUSSION}

There was zero hatching success at 6,12 and 18 hours under all treatments. Daphnia magna eggs exposed to uncontaminated sediment hatch between 18 and 24 hours (time zero taken as the attainment of the single median eye stage of egg development).

Hatching success was significantly reduced by the presence of the spiked mixture of heavy metal salts (Fig 1). Hatching success was $100 \%$ in uncontaminated sand and declined to $0 \%$ at the highest concentrations of metal salts. Similarly, in the AMD contaminated sediments (Fig 2), hatching success was $100 \%$ in uncontaminated sands and declined to $0 \%$ in the most contaminated (10\% AMD sediments). Thus the test effectively detects contaminated AMD sediments at intermediate levels of contamination, which is an important attribute of for testing along the length of a watershed. One advantage of the proposed test is the speed at which contaminant toxicity is determined. This can help managers to effectively address the impact of pollutants that have short in-stream residence times. The sensitivity of the embryonic tests over tests that use adult life stages is another potential advantage of this test. We expect that this test would be generally applicable to determining the toxicity of AMD pollutants, as well as other complex mixtures of pollutants.

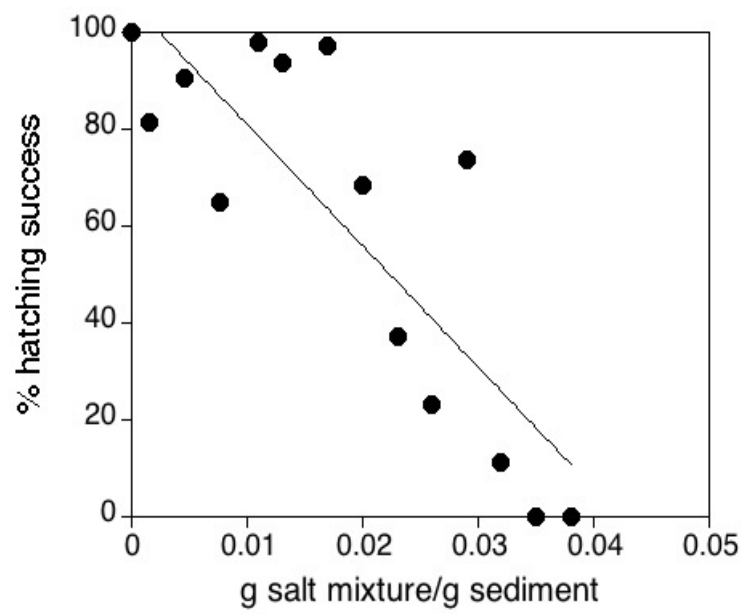

Fig 1. Hatching success in Daphnia magna exposed to increasing concentrations of metallic salt mixtures in spiked sediment $(\mathrm{p}=$ $0.0002, r=-0.83)$

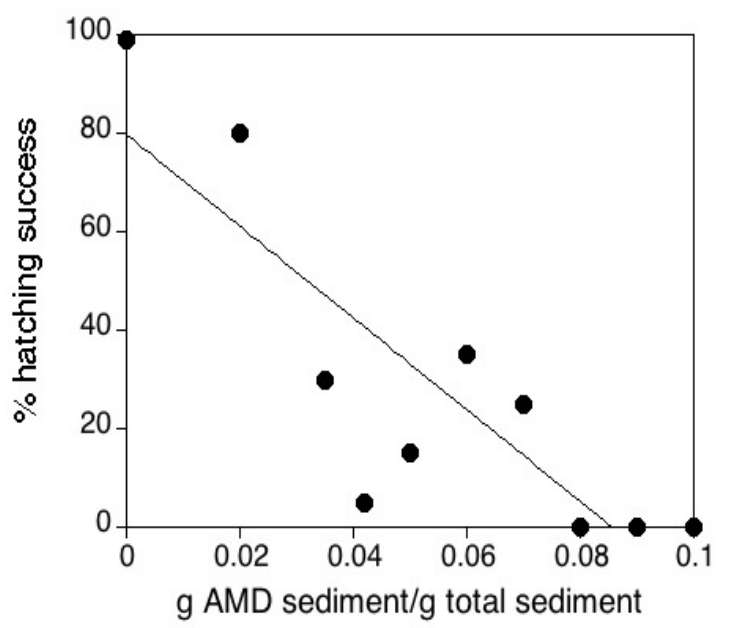

Fig 2. Hatching success in Daphnia magna exposed to increasing proportions of AMD sediments to clean sands $(\mathrm{p}=0.02, \mathrm{r}=-$ $0.74)$.

Acknowledgements: This research was supported by an Ohio University Research Council award to R. P. Hassett.

\section{REFERENCES}

ASTM (2006): E 1193-97 Standard Guide for Conducting Daphnia magna Life-Cycle Toxicity Tests. Annual Book of Standards. Vol. 11.05 Biological Effects and Environmental Fate; Biotechnology; Pesticides. ASTM International, West Conshohocken, PA

Baudo, R (1987) Ecotoxicological Testing with Daphnia. Mem Ist Ital Idrobiol 45: 461-682 
Dave, G (1993). Replicability, repeatability, and reproducibility of embryo larval toxicity tests with fish. In Progress in Standardization of aquatic Toxicity Tests (A M V M Soares and P Calow, Eds.), 129-157. Lewis, Boca Raton, FL.

D'Sa, J V (2004). Rapid Removal of Toxicity of Acid Mine Drainage Contaminated Sediments in a Clean Stream: Relative Contribution of Acidity Vs Metals. Masters Thesis, Ohio University

Lopes, I; Soares, A M V M; Ribeiro, R (1999). Discriminating the Ecotoxicity Due to Metals and to Low $\mathrm{pH}$ in Acid Mine Drainage. Ecol Environ Safety 44: 207-214

Martinez-Tabche, L; Gomez-Olivan, L; Martinez, M G; Castillo, C R; Santiago, A (2000). Toxicity of Nickel in Artificial Sediment on Acetylcholinesterase Activity and Hemoglobin Concentration of the Aquatic Flea, Moina macrocopa. J Environ Hydrol 8: Paper 4

Martins, J; Oliva Teles, L; Vasconcelos, V (2007) Assays with Daphnia magna and
Danio rerio as alert systems in aquatic toxicology. Environ Intern 33: 414-425

Pereira, A M M; Soares, A M V M; Goncalves, F; Ribeiro, R (2000). Water-Column, Sediment, and in Situ Chronic Bioassays with Cladocerans. Ecotox Environ Safety 47: $27-38$

Smith, E.E.; K.S. Shumate. (1970). Sulfide to sulfate reaction mechanism. Water Poll Control Res Ser 14010 FPS. USDI, FWQA. Washington, D.C.

Sobral, O; Chastinet, C; Nogueira, A; Soares, A M V M; Goncalves, F; Ribeiro, R (2001). In Vitro Development of Parthenogenetic eggs: A Fast Ecotoxicity Test With Daphnia magna? Ecotox Environ Safety 50: 174$179 \mathrm{~b}$

United States Environmental Protection Agency (USEPA) (2000). Methods for measuring the toxicity and bioaccumulation of sediment-associated contaminants with freshwater invertebrates, Second Edition (Report EPA 600/R-99/064) 